\title{
IFIH1-GCA-KCNH7 locus: influence on multiple sclerosis risk
}

\author{
Alfonso Martínez ${ }^{1}$, José Luis Santiago ${ }^{1}$, M Carmen Cénit $^{1}$, Virginia de las Heras ${ }^{2}$, \\ Hermenegildo de la Calle $^{3}$, Miguel Fernández-Arquero ${ }^{1}$, Rafael Arroyo ${ }^{2}$, Emilio G de la \\ Concha $^{1}$ and Elena Urcelay ${ }^{\star 1}$
}

${ }^{1}$ Department of Immunology, Hospital Universitario San Carlos, Madrid, Spain; ${ }^{2}$ Department of Neurology, Hospital
Universitario San Carlos, Madrid, Spain; ${ }^{3}$ Department of Endocrinology, Hospital Ramón y Cajal, Madrid, Spain

A recent genome-wide scan of nonsynonymous SNPs and ulterior validation in case-control and family analyses evidenced a susceptibility locus for type 1 diabetes (T1D) on chromosome 2 q24.3. We aimed at testing the effect of this locus in other autoimmune diseases with complex genetic background, such as multiple sclerosis (MS). Four SNPs along the locus, rs13422767, rs2111485, rs1990760 and rs2068330, were genotyped using TaqMan MGB chemistry in 311 T1D and 412 MS patients and 535 ethnically matched healthy controls. The previously reported association of this locus was found for the first time in MS (rs2068330, G vs C: $P=0.001 ;$ OR $(95 \% \mathrm{Cl})=0.73(0.6-0.88)$ ) and a trend for replication was observed in our Spanish diabetic cohort. Therefore, genes included in this locus - IFIH1 interferon induced helicase, GCA grancalcin or the potassium channel $\mathrm{KCNH7}$ - are potential candidates implicated in the pathogenesis of these autoimmune diseases, although strong linkage disequilibrium in the region hampered further localization of the etiologic gene. European Journal of Human Genetics (2008) 16, 861-864; doi:10.1038/ejhg.2008.16; published online 20 February 2008

Keywords: IFIH1 interferon induced helicase; GCA grancalcin; KCNH7 potassium channel; multiple sclerosis; T1D disease susceptibility

\section{Introduction}

Determination of the etiology of immune-mediated diseases as multiple sclerosis (MS) remains largely elusive, although numerous theories have been proposed. Environmental factors operate on a background of genetic susceptibility in its pathogenesis. MS incidence in many countries has risen rapidly over the past decades, suggesting increased environmental pressure on susceptibility genotypes.

With regard to the environmental factors involved, viral infections have been considered in these diseases. ${ }^{1}$ Innate immune responses to pathogens are initiated after the

*Correspondence: Dr E Urcelay, Department of Immunology, Hospital Universitario San Carlos, Martin Lagos s/n, 28040 Madrid, Spain.

Tel: + 34-91 330 3347; Fax: + 34-91 330 3344;

E-mail: eurcelay.hcsc@salud.madrid.org

Received 24 October 2007; revised 21 December 2007; accepted 11 January 2008; published online 20 February 2008 recognition of so called pathogen-associated molecular patterns by cellular receptors. ${ }^{2}$ Among these receptors, the cytoplasm helicase MDA-5 senses intracellular dsRNA resulting from viral infection. The gene encoding MDA-5 (melanoma differentiation-associated gene 5, MDA5) also known as interferon-inducible RNA helicase IFIH1 is located in chromosomal region $2 \mathrm{q} 24$.3. A common variant in the IFIH1 locus has been recently found associated with type 1 diabetes (T1D) in a robust study. ${ }^{3}$ This genome-wide association study and ulterior validation in a case-control and family collection evidenced the involvement of rs1990760, an Ala946Thr change in the IFIH1 gene, in T1D risk. Further analyses of the locus surrounding this marker yielded compelling statistical support of the genetic quasi-equivalence of some other SNPs in strong linkage disequilibrium with the above-mentioned polymorphism, therefore being unable to ascertain the etiologic variant. 
The other genes mapping within the associated region, the voltage-gated potassium channel $\mathrm{KCNH} 7$ and the grancalcin GCA genes, are both potential candidate genes from a functional standpoint. Voltage-dependent $\mathrm{K}^{+}$channels constitute a heterogeneous class of ion channels including more than 40 members. ${ }^{4}$ Among voltage-dependent $\mathrm{K}^{+}$ channels, the ERG subfamily is mainly involved in the control of cellular excitability, both in mammals and in invertebrates. ${ }^{5,6}$ Moreover, macrophages and neutrophils are key cellular components of innate immunity and the GCA grancalcin gene, located between IFIH1 and $\mathrm{KCNH7}$ genes, is highly expressed in both types of leukocytes. ${ }^{7}$

More recently, association of this locus with Grave's disease was also found. ${ }^{8}$ We aimed at identifying whether this chromosomal region was a common susceptibility locus for other autoimmune disease as MS.

\section{Methods}

Three hundred and eleven white Spanish unrelated T1D (51\% women) and 412 MS (65\% women) patients consecutively recruited from a single center (Hospital Clínico, Madrid) and 535 ethnically matched healthy controls (51\% women), mainly blood donors and staff from the same region, were included in a case-control study. T1D patients were diagnosed according to the American Diabetes Association (ADA) criteria. MS diagnosis conformed to the Poser criteria ${ }^{9}$ and $36 \%$ MS patients carried HLA-DRB1*1501. Most patients were relapsing remitting (79\%), 11\% secondary progressive and 9\% primary progressive. Informed consent was obtained from all the subjects included in the study, which was approved by the Ethics Committee of the Hospital Clínico San Carlos.

Genotyping of the samples was performed by using TaqMan Assays in a 7900HT Fast Real-Time PCR system under the conditions recommended by the manufacturer (Applied Biosystems, Foster City, CA, USA).

Statistical analyses were performed using a standard statistical package (Epi Info v.6.02; World Health Organization, Geneva, Switzerland). Phenotype and genotype frequencies in patients and controls were compared by $\chi^{2}$ test or Fisher's exact test, when necessary. Strength of association was given as odds ratio (OR) with a 95\% confidence interval (CI) and $P$-values under 0.05 were considered statistically significant. Haplotypic frequencies were estimated using the expectation-maximization algorithm implemented in the Arlequin v2.000 software, ${ }^{10}$ with number of iterations set at 5000 and initial conditions at 50, with an epsilon value of $10^{-7}$.

\section{Results}

Four polymorphisms within the chromosomal region originally found associated with T1D were studied in the Spanish population: rs1990760, in exon 15 of the IFIH1 gene, and two additional SNPs in the $5^{\prime}$ and $3^{\prime}$ boundaries of the associated locus: rs13422767 and rs2068330, the latter in intron 14 of the $K C N H 7$ gene. The intergenic polymorphism rs13422767 did not conform to HardyWeinberg expectations in the control cohort $(P<0.05)$; therefore, it was excluded from ulterior analyses and replaced by other in proximity, rs2111485, which did not show significant difference between patients and controls (Table 1). Minor alleles of the two other polymorphisms evidenced protective effects towards MS of similar magnitude to the one originally reported by Smyth et $a l^{3}$ and a trend for T1D patients, most probably due to lower statistical power (Table 1). Due to strong linkage disequilibrium between those two associated SNPs in the IFIH1 and in the KCNH7 genes $\left(\mathrm{D}^{\prime}=0.81 ; r^{2}=0.58\right)$ we could not ascertain which, if any, was primarily associated with the diseases. However, in our hands, rs2068330 behaved as a better marker from a statistical point of view and for practical purposes it was chosen for sub-group analyses. Genotype relative risks were calculated for the heterozygous (T1D: $P=0.8$; OR $(95 \% \mathrm{CI})=0.95(0.7-1.3)$ and MS: $P=0.004$; OR $(95 \% \mathrm{CI})=0.67(0.5-0.89))$ and homozygous mutant (T1D: $P=0.03$; OR $(95 \% \mathrm{CI})=0.6(0.36-0.99)$ and MS: $P=0.009$; OR $(95 \% \mathrm{CI})=0.58(0.37-0.89))$ categories. Similar effects could be detected for both genotypes in MS and for the homozygous mutant in T1D, and the lower effect displayed by the heterozyote in T1D is most probably due to scarce statistical power of this cohort. Haplotype analysis did not provide any additional information (data not shown). Stratification of MS patients by clinical forms and by carriage of HLA-DRB1*1501 did not show statistical differences.

Table 1 Genotype and allele frequencies of three SNPs in the 2q24.3 chromosomal region containing the IFIH1-GCA-KCNH7 genes

\begin{tabular}{|c|c|c|c|c|c|c|c|c|c|c|c|c|c|c|c|}
\hline & \multicolumn{5}{|c|}{ rs2111485 (\%) } & \multicolumn{5}{|c|}{ IFIH1, rs1990760 (\%) } & \multicolumn{5}{|c|}{ KCNH7, rs2068330 (\%) } \\
\hline & $G G$ & $G A$ & $A A$ & $G$ & $A$ & $A A$ & $A G$ & GG & $A$ & G & $\mathrm{CC}$ & $C G$ & $G G$ & $C$ & $G$ \\
\hline Controls & 191 & 250 & 86 & 632 (59.9\%) & 422 (40\%) & 188 & 254 & 93 & $630(58.9 \%)$ & $440(41.1 \%)$ & 204 & 252 & 79 & $660(61.7 \%)$ & 410 (38.3\%) \\
\hline MS patients & 163 & 175 & 61 & $501(62.8 \%)$ & $297(37.2 \%)$ & 170 & 183 & 62 & $523(63 \%)$ & 307 (37\%) & 201 & 166 & 45 & $568(68.9 \%)$ & $256(31.1 \%)$ \\
\hline
\end{tabular}

rs2111485, A vs G: T1D: $P=0.42 ; \mathrm{OR}=0.92$ (0.75-1.13), MS: $P=0.22 ; \mathrm{OR}=0.89(0.73-1.08)$.

rs1990760, G vs A: T1D: $P=0.11 ; \mathrm{OR}=0.85(0.7-1.04), \mathrm{MS}: P=0.07 ; \mathrm{OR}=0.84(0.7-1.02)$.

rs2068330, G vs C: T1D: $P=0.08 ; \mathrm{OR}=0.83(0.67-1.03), \mathrm{MS}: P=0.001 ; \mathrm{OR}=0.73(0.6-0.88)$. 


\section{Discussion}

In the present work, we investigated the association of polymorphisms within the locus where the IFIH1-GCAKCNH7 genes map with MS. The effect found for MS was of similar magnitude to the one originally described regarding $\mathrm{T}^{1} \mathrm{D}^{3}$ and also present in Graves' disease. ${ }^{8}$ Strong linkage disequilibrium within this locus precluded us from further dissecting where the etiologic gene resides. The polymorphism rs1990760 in the IFIH1 gene was proposed as the causal variant based on the conservation of aligned sequences in mammals. ${ }^{3}$ However, the authors reported that this polymorphism was genetically equivalent to some others and it was indistinguishable from the other tested variant in the $\mathrm{KCNH} 7$ gene. Intronic polymorphisms, as the one in the $K C N H 7$ gene, could also be implicated in the disease pathogenesis through altered splicing. ${ }^{11}$ As a recent report demonstrated, even naturally occurring variations in synonymous codons can give rise to a protein product with the same amino-acid sequence but different structural or functional features, probably due to altered timing of cotranslational folding. ${ }^{12}$ Consequently, polymorphisms that do not change coding sequences should not be disregarded as contributors to disease.

From a functional perspective, it is difficult to ascertain which of these genes would be the one responsible for the observed associations. Many human viruses have been associated with autoimmune complex conditions. The recognition that the pre-clinical phase of autoimmunity may extend for years is critical to understanding their etiology and means that studies at onset may not reveal viral infections. Moreover, a common virus could act as a trigger in genetically susceptible hosts and if infections are due to ubiquitous viruses, differences in the frequency of infections will not be detectable in case-control studies. As a study of children developing T1D showed, the majority of seasonality at onset was in the non-DR3/4 males, suggesting that viral infection could be an additive risk factor in those less genetically predisposed. ${ }^{13}$ The interferon-inducible helicase IFIH1 gene encodes a cytoplasmic viral detector critical for response to picornaviruses. ${ }^{14}$ Picornavirus infections have been associated with $\mathrm{MS}^{15,16}$ and T1D. ${ }^{17-19}$ Studies, in vitro and in vivo, demonstrated that human pancreatic $\beta$ cells survive challenge with coxsackie virus only in the presence of interferons and that protection from diabetes following systemic infection required an intact islet cell response to interferons. ${ }^{20,21}$ These evidences, together with the work by Smyth et al, ${ }^{3}$ the association reported in Graves' disease ${ }^{8}$ and our own data, would support interferon-inducible helicase IFIH1 as a candidate gene altering susceptibility to autoimmune diseases. Nonetheless, in our hands the polymorphism within the $K C N H 7 / E R G 3$ gene seems to act as a better genetic maker and an evidence in support of the impact of potassium homeostasis in these diseases has also been reported. Voltage-dependent $\mathrm{K}^{+}$channels are the main determinants in controlling cellular excitability within the central nervous system; specifically, the erg genes are highly expressed in various areas of the rat and mouse central nervous system ${ }^{22}$ and $\operatorname{erg} 3$ transcripts have also been identified in rat islets and INS-1 $\beta$ cells. ${ }^{23}$ Therefore, implication on autoimmune diseases of an altered function of this gene encoding a potassium channel can not be discarded. Not only these two, but the other positional candidate gene, the one encoding grancalcin, could also have a role in autoimmune disease pathogenesis. The GCA protein presents a calcium binding EF-hand domain characterizing a large family thought to play a pivotal role in a variety of signaling pathways. ${ }^{24}$

In summary, we report for the first time the association of the IFIH1-GCA-KCNH7 locus with MS. It could be speculated that any of the genes within the associated chromosomal region could be the one responsible for the observed effect; replication in populations of diverse ethnicities, in which linkage disequilibrium patterns may be dissimilar, are of crucial importance to further localize the etiologic gene involved.

\section{Acknowledgements}

We thank Carmen Martínez for her skilful technical assistance. Alfonso Martinez and Jose Luis Santiago are recipients of FIS contracts (CP04/00175 and CM05/00216, respectively). Elena Urcelay works for the 'Fundación para la Investigación Biomédica-Hospital Clínico San Carlos'. This work was supported by grants from: FIS PIO70369, FIS PIO70353 and FIS PI051221.

\section{References}

1 Fujinami RS, von Herrath MG, Christen U, Whitton JL: Molecular mimicry, bystander activation, or viral persistence: infections and autoimmune disease. Clin Microbiol Rev 2006; 19: 80-94.

2 Janeway Jr CA, Medzhitov R: Innate immune recognition. Annu Rev Immunol 2002; 20: 197-216.

3 Smyth DJ, Cooper JD, Bailey R et al: A genome-wide association study of nonsynonymous SNPs identifies a type 1 diabetes locus in the interferon-induced helicase (IFIH1) region. Nat Genet 2006; 38: $617-619$.

4 Gutman GA, Chandy KG, Grissmer S et al: International Union of Pharmacology. LIII. Nomenclature and molecular relationships of voltage-gated potassium channels. Pharmacol Rev 2005; 57: 473-508.

5 Furlan F, Guasti L, Avossa D et al: Interneurons transiently express the ERG $\mathrm{K}+$ channels during development of mouse spinal networks in vitro. Neuroscience 2005; 135: 1179-1192.

6 Guasti L, Cilia E, Crociani O et al: Expression pattern of the ethera-go-go-related (ERG) family proteins in the adult mouse central nervous system: evidence for coassembly of different subunits. J Comp Neurol 2005; 491: 157-174.

7 Liu F, Shinomiya H, Kirikae T, Hirata H, Asano Y: Characterization of murine grancalcin specifically expressed in leukocytes and its possible role in host defense against bacterial infection. Biosci Biotechnol Biochem 2004; 68: 894-902.

8 Sutherland A, Davies J, Owen CJ et al: Genomic polymorphism at the interferon-induced helicase (IFIH1) locus contributes to Graves' disease susceptibility. J Clin Endocrinol Metab 2007; 92: 3338-3341.e-pub 2007 May 3329. 
9 Poser CM, Paty DW, Scheinberg L et al: New diagnostic criteria for multiple sclerosis: guidelines for research protocols. Ann Neurol 1983; 13: 227-231.

10 Excoffier L, Slatkin M: Maximum-likelihood estimation of molecular haplotype frequencies in a diploid population. Mol Biol Evol 1995; 12: 921-927.

11 Law AJ, Kleinman JE, Weinberger DR, Weickert CS: Diseaseassociated intronic variants in the ErbB4 gene are related to altered ErbB4 splice-variant expression in the brain in schizophrenia. Hum Mol Genet 2007; 16: 129-141.

12 Kimchi-Sarfaty C, Oh JM, Kim IW et al: A 'silent' polymorphism in the MDR1 gene changes substrate specificity. Science 2007; 315: $525-528$.

13 Weets I, Kaufman L, Van der Auwera B et al: Seasonality in clinical onset of type 1 diabetes in belgian patients above the age of 10 is restricted to HLA-DQ2/DQ8-negative males, which explains the male to female excess in incidence. Diabetologia 2004; 47: 614-621.

14 Kato H, Takeuchi O, Sato S et al: Differential roles of MDA5 and RIG-I helicases in the recognition of RNA viruses. Nature 2006; 441: $101-105$.

15 Kriesel JD, White A, Hayden FG, Spruance SL, Petajan J: Multiple sclerosis attacks are associated with picornavirus infections. Mult Scler 2004; 10: $145-148$.

16 Olson JK, Ercolini AM, Miller SD: A virus-induced molecular mimicry model of multiple sclerosis. Curr Top Microbiol Immunol 2005; 296: 39-53.
17 Benoist C, Mathis D: Autoimmunity provoked by infection: how good is the case for T cell epitope mimicry? Nat Immunol 2001; 2 797-801.

18 Skarsvik S, Puranen J, Honkanen J et al: Decreased in vitro type 1 immune response against coxsackie virus B4 in children with type 1 diabetes. Diabetes 2006; 55: 996-1003.

19 Yoon JW, Jun HS: Viruses cause type 1 diabetes in animals. Ann NY Acad Sci 2006; 1079: 138-146.

20 Chehadeh W, Kerr-Conte J, Pattou F et al: Persistent infection of human pancreatic islets by coxsackievirus $\mathrm{B}$ is associated with alpha interferon synthesis in beta cells. J Virol 2000; 74: $10153-10164$

21 Flodstrom M, Maday A, Balakrishna D, Cleary MM, Yoshimura A, Sarvetnick N: Target cell defense prevents the development of diabetes after viral infection. Nat Immunol 2002; 3: 373-382.

22 Restano-Cassulini R, Korolkova YV, Diochot S et al: Species diversity and peptide toxins blocking selectivity of ether-a-gogo-related gene subfamily $\mathrm{K}+$ channels in the central nervous system. Mol Pharmacol 2006; 69: 1673-1683.

23 Muhlbauer E, Bazwinsky I, Wolgast S, Klemenz A, Peschke E: Circadian changes of ether-a-go-go-related-gene (Erg) potassium channel transcripts in the rat pancreas and beta-cell. Cell Mol Life Sci 2007; 64: 768-780.

24 Nacken W, Sorg C, Kerkhoff C: The myeloid expressed EF-hand proteins display a diverse pattern of lipid raft association. FEBS Lett 2004; 572: 289-293. 